\title{
Biophysical methods: behind the scenes of the cryo-EM revolution
}

\author{
Carla Schmidt ${ }^{1}$ and Carol V. Robinson ${ }^{2}$ \\ ${ }^{1}$ Interdisciplinary research center HALOmem, Martin Luther University Halle- \\ Wittenberg, Halle, Germany \\ ${ }^{2}$ Department of Chemistry, Physical \& Theoretical Chemistry Laboratory, University \\ of Oxford, Oxford, United Kingdom
}

During the preparation of this issue two significant events took place. First the sad passing of Anna Tramontano, after suffering a short illness and following the submission of her article. Her contributions to the field of computational prediction of protein assemblies are well-documented and her final article, presented in this issue, serves as a fitting memorial to her recent opinion of the field. The second event was the award of the Nobel Prize for Chemistry for the development of Cryo-electron microscopy (Cryo-EM). We celebrate the life of Anna Tramontano and congratulate the three recipients of the prize. This collection of articles is therefore both a fitting tribute to Anna as well as a celebration of the dramatic advances made in Cryo-EM, in its own right, and in combination with other biophysical approaches.

The many beautiful high-resolution structures emanating from Cryo-EM have been widely publicised with each new structure greeted with enthusiasm and reviewed widely elsewhere. Rather than reproduce these high profile structures we focus this series of reviews on the unseen heroes of the so called 'resolution revolution', the activists behind the scenes, that contribute to the deduction of these amazing structures.

Starting with the 'nuts and bolts' of cryo-EM itself Elmlund et al. provide an in-depth description of the underlying processes from specimen preparation and stabilisation on the grid, through to consideration of motion correction and electron damage limitation. Parallel developments in electron cryo-tomography (cryoET) have also taken advantage of technological innovation, specifically the direct electron detectors from cryo-EM (Baker et al.). For cryo-ET frozen-hydrated samples are used to determine the structure of macromolecular complexes in their native environment with the overall goal of visualizing cellular features and structures of macromolecular complexes in situ. A great strength of cryoET is the diversity of preparations it can accommodate, from cells to reconstituted protein complexes.

While the size of protein complex amenable to study by cryoEM is steadily 
decreasing accessing protein dynamics using this approach remains challenging and, as mentioned above, motion correction is often required to enhance imaging. As such alternative methods are required to address small molecule motion. Typically Förster (or fluorescence) resonance energy transfer (FRET) has been exploited for this purpose in various in-cell measurements and single molecule methods. The socalled 'FRET nanoscopic ruler' can measure distances between donor and acceptor molecules with great precision informing the motion of interacting partners. Recent developments reviewed here (Okamoto and Sako) include a quantitative evaluation of protein interactions and protein structures wherein different conformers are assigned based upon their FRET signature. A particular limitation arises when conformers with the same FRET cannot be distinguished. One possible solution to this problem recently implemented is to apply molecular dynamics simulations to obtain FRET distributions of fluctuating molecules in solution.

Continuing with the theme of FRET Danial et al. summarize notable breakthroughs in biological research based on single molecule imaging, identifying shortcomings in single molecule detection and presenting current opinion on ameliorating some of its limitations for wider applicability. Localizing single molecules in the structure of the nuclear pore complex provides a stunning example of how counting, monitoring, localizing and tracking single molecules have become feasible, shedding new light on the structure and function of biological systems. In spite of its merits, the utility of small molecule FRET as a 'nanoscopic ruler' comes with plenty of challenges many of which ammelerioted by careful choice of appropriate residues for attaching fluorophore pairs and by distinguishing compaction due to crowding from conformational change.

Similar to the nanoscale ruler employed in FRET experiments is the chemical crosslinking approach utilised in conjunction with mass spectrometry (CX-MS) (Schmidt and Urlaub). By covalently linking proximal amino-acid residues, digestion of the complex and assignment of the crosslinked peptides, distance constraints can be obtained for homology or de novo modelling. Combining cryo-EM with CX-MS enables one to probe the inaccessibility of the interior and to probe regions that are less well-defined. Moreover conformational changes in protein complexes can be deduced through comparative crosslinking strategies in which chemically labeled cross-linkers are used to report on macromolecular complexes in different scenarios. 
ensemble of oligomeric species present at equilibrium Hoffmann and colleagues describe the application of ion mobility MS to the determination of intermediates in amyloid fibril formation. As is often the case using biophysical approaches in combination provides unexpected synergies and here by means of an innovative application of infra red spectroscopy, in combination with ion mobility MS, the authors demonstrate how they can define secondary structure content of the various oligomers formed. Probing a series of intermediates populated during $A \beta$ fibril formation they demonstrate that from tetramers to nonamers beta sheet content is preserved.

With the excitement of the Cryo-EM advances it is important not to forget X-ray crystallography for deducing those parts of the complex that are not readily defined in cryo-EM (Lea and Higgins). Still at the heart of integrative structural biology many recent novel crystal structures have revealed with unprecedented detail mechanisms by which antibodies and proteins from tick salivary glands for example can inhibit critical complement pathways. Membrane protein crystal structures, often considered the most challenging of targets, continue to emerge from various membrane mimetics while the bright lights of the $\mathrm{X}$-ray free electron lasers are beckoning the structural biology community with the promise of high-resolution structures without crystals.

To capitalize on all these approaches and to fully characterize the heterogeneity of composition and conformational states of protein assemblies integrative modelling of cellular assemblies, including protein complexes and genomic assemblies remains critical (Joseph et al.). Recent examples of integrative models generated for such assemblies by different experimental techniques, include data from 3D electron microscopy (3D-EM). The authors highlight general concepts for consideration in integrative modelling and discuss the need for careful formulation and merging of different types of information. The authors caution that the final model(s) should also reflect uncertainty and completeness of input information and advise cross-validation against an independent data set for model assessment and the detection of overfitting. Large conformational variability in cellular assemblies often affects the consistency between different experimental data and this requires generation of an ensemble of models to more accurately represent the data. The authors call for new community efforts to address issues relating to the standardization of representation, validation and archiving of integrative models. 
As an alternative approach computer prediction of protein complexes continues to be an active area of research. Tramontano states the problem clearly as that of identifying partners, which is usually addressed using sequence and network based approaches, while the prediction of the actual structure of complexes is mainly addressed by structure based (docking) and/or by evolutionary based methods. She points out the merits of sequence and network-based approaches and states that they cannot easily disentangle functional interactions, those that have a biological effect, from logical ones without physical contact. Having identified associations therefore, subsequent analyses are required to assess whether the interaction is a true physical one. The author then discusses the evaluation data of the last Critical Assessment of Techniques for Protein Structure Prediction (CASP12). She reports that of the thirty CASP12 targets, that match in size and symmetry the distribution of multimeric proteins in the PDB, very few groups predicted more than half of the targets. She concludes that different methods are appropriate for different cases and that universal approach is not yet forthcoming

Bringing all biophysical techniques to bear on a problem, with computational support, is clearly the future. Highlighting the potential of a powerful combination of in-cell NMR, cryo-ET and in-cell single molecule FRET to move into cellular structural biology lies at the heart of the vision of the contribution from Plitzko, Schuler and Selenko. Making the case for integrated use of complementary single- and ensemble-molecule methods operating at different levels of resolution for comprehensive in-cell studies in years to come. The authors call for community wide efforts to integrate these techniques with a particular focus on the need to make measurements in biologically relevant cell types and with a call for training programs at the interface of cell biology and biophysics. The authors claim that while many laboratory in situ approaches exploit generic, 'easy to handle' cell lines these fall short of representing the most suitable biological context. Primary cells or dissected tissue sections are the ultimate goal but are not yet feasible for techniques that require large numbers of molecules for study such as NMR. For such approaches the use of pluripotent stem cells, and their ability to differentiate into specialized cellular lineages, offers appealing alternative for the future. For the time being measurements in cells are inherently noisy but as we move forward into the new era of high resolution cryo-EM and cryo-ET, in combination with other approaches, the goal of structural biology in the cell becomes no longer a pipe dream but a distant reality, likely to be realized within the next decade. 
